\title{
ISENTROPIC EFFICIENCY OF CENTRIFUGAL COMPRESSOR WORKING WITH REAL GAS
}

\begin{abstract}
JIŘÍ OLDŘICH
Howden ČKD Compressors s.r.o., Klečákova 347/5, 190 00, Praha 9, Czech Republic

correspondence: jiri.oldrich@howden.com, oldrich.jiri@seznam.cz

Abstract. The contribution deals with calculation of isentropic efficiency and also with calculation of isentropic process of real gas or gaseous mixtures. The method is based on numerical solution of basic definitional equation of isentropic process and equation of isentropic efficiency with direct implementation of real gas equation of state (EOS).
\end{abstract}

KEYWORDS: Efficiency, isentropic, real gas, compressor.

\section{INTRODUCTION}

Isentropic efficiency is an important parameter widely used in thermodynamic design of centrifugal compressor. Reversible adiabatic process identical to isentropic process is used as reference process. The isentropic process is a process to which great attention is devoted in literature. In some articles certain inaccuracies are used repeatedly. For example dependence of heat capacities on temperature is ignored. Very often Poisson equations are used for calculation of real gases. Isentropic exponents that are generally dependent on temperature and pressure are often substituted by mean values. Adiabatic and isentropic processes are considered synonymous. These inaccuracies can generate noticeable errors in calculations.

The aim of this contribution is to clarify the above mentioned inaccuracies and to suggest thermodynamically correct procedures that are appropriate for PC's. In the first part of contribution equation for some simple special cases is described very shortly. The second more extensive part deals with techniques for various combinations of input and output variables. All these techniques are based on behavior of real gases and are independent on real gases EOS used.

\section{ISENTROPIC EFFICIENCY}

Efficiency of compressor that does not exchange heat with the environment (compressor without intercooling or uncooled compressor section) is calculated by comparing the work that was actually spent on compression and the appropriate reference process. Reference process is usually, depending on the compression process in the compressor, an isentropic process [14], a polytropic process [2, 3, 5, 6, or a reversible isothermal process [2, 3, 7]. We will only deal with a reversible isentropic process and isentropic compression in the following text.

In the actual process that occurs in the compressor, losses occur in the flowing gas and, as a result of it, some part of the kinetic energy is converted into heat. This process leads to an increase in temperature and

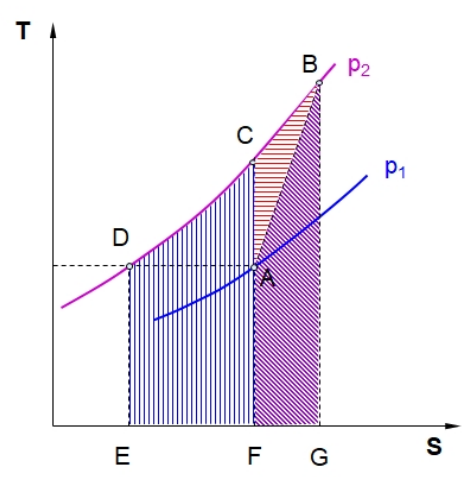

Figure 1. Isentropic (area $A C D E F A$ ) and actual work (area $G B C D E F G$ ) in $T$-S diagram when compressed from pressure $p_{1}$ to pressure $p_{2}$.

entropy of compressed gas. A small part of the generated heat is discharged from the compressor casing to the surroundings. The process that is going on in the actual compressor is irreversible. The final temperature of the gas after its compression is always higher than temperature of gas after isentropic compression. In the diagram $H-S$ in FIGURE 1 the reversible isentropic compression work is represented by the area $A C D E F A$ and the actual work spent on compression from pressure $p_{1}$ to the pressure $p_{2}=p_{2 a}=p_{2 s}$ is represented by the area $G B C D E F G$. The work actually spent is irreversible and can be divided into isentropic work, compression losses (area $A B G F A$ ) and increase of compression work due to losses (area $A B C A$ )

$$
\Delta H_{a}=H_{2 a}-H_{1} .
$$

Isentropic efficiency is defined as a relation between reversible isentropic work and actual work

$$
\eta_{s}=\frac{\Delta H_{s}}{\Delta H_{a}}=\frac{H_{2 s}-H_{1}}{H_{2 a}-H_{1}} .
$$

Short repetition of basic principles [2, 3, 8].

- Adiabatic (isentropic) efficiency does not depend only on amount of losses. It depends on amount 


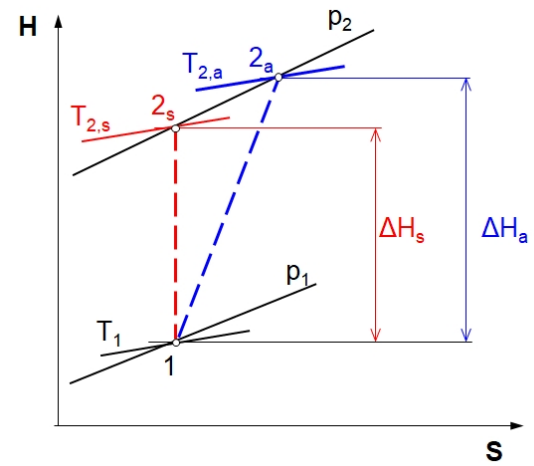

Figure 2. Isentropic and actual processes in $H-S$ diagram.

of additional increase in compression work due to losses expressed by the area $A C B A$, too.

- There are losses in gas flow in the actual process that occurs in compressor, and as a result, a part of the kinetic energy is converted into thermal energy and hence an increase in temperature and entropy of compressed gas.

- At the same quality of compression mean value of additional increasing in compression work increases with increasing of total pressure.

- At the same quality of compression the adiabatic efficiency will be at higher pressure ratio lower than at lower pressure ratio.

- Temperature of gas after actual compression is always higher than temperature after isentropic compression (see FIGURE 2).

\section{Equation of isentrope}

Adiabatic process is defined by the equation

$$
d q=0
$$

where $q$ is heat input into system

$$
d S=\frac{d q}{T}=0
$$

Reversible adiabat is identical to isentrope. In irreversible process is

$$
d S>\frac{d q}{T}=0
$$

Adiabat and isentrope are not identical. We rewrite equation (4) into form

$$
\begin{aligned}
d S=\left(\frac{\partial S}{\partial T}\right)_{V} d T & +\left(\frac{\partial S}{\partial V}\right)_{T} d V= \\
& =\frac{C_{V}}{T} d T+\left(\frac{\partial p}{\partial T}\right)_{V} d V=0
\end{aligned}
$$

where $p$ is the pressure of system and $C_{V}$ is heat capacity, which is derivative of internal energy with respect to temperature at constant volume

$$
C_{V}=\left(\frac{\partial U}{\partial T}\right)_{V} .
$$

That generally depends on temperature and on volume. For ideal gas the heat capacity $C_{V}=C_{V}^{0}$ depends only on temperature.

Equation of isentrope is obtained by integrating the relationship (6). Its specific form depends on the chosen equation of state from which we determine $(\partial p / \partial T)_{V}$ and the chosen equation for the temperature dependence of $C_{V}^{0}$.

\section{ReVERsible process, IDEAL GAS, CONSTANT $C_{V}^{0}$ (PERFECT GAS)}

This simplest case leads to the known Poisson equations. Equation (6) we rewrite into form

$$
\frac{C_{V}^{0}}{T} d T=-\frac{R}{V} d V
$$

where derivative $(\partial p / \partial T)_{V}$ is found from equation of state written for one mole

$$
p V=R T
$$

After integration of Eq. (8) and after modification we receive well known Poisson equation in variables $T-V$

$$
T V^{k-1}=\text { const. }
$$

where $k=\left(C_{V}^{0}+R\right) / C_{V}^{0}$. Poisson equations in variables $T-p$ and $p-V$ we obtain by inputting of volume $V$ or temperature $T$ from equation of state $(9)$

$$
\begin{gathered}
T p^{\frac{1-k}{k}}=\text { const. } \\
p V^{k}=\text { const. }
\end{gathered}
$$

\section{Reversible process, General EQUATION OF ISENTROPE}

From Eq. 4 we obtain general equation of isentrope

$$
S(T, V)=\text { const }
$$

Then the problem is reduced to standard calculation of entropy for known (chosen) equation of state and equation of temperature dependence of $C_{V}^{0}$ (Figure 3). In the following text we will use the molar density $\rho=1 / V$ instead of the volume as an independent variable. We will write the equation of the isentrope in the form

$$
S\left(T_{2}, \rho_{2}\right)-S\left(T_{1}, \rho_{1}\right)=0
$$

where index 1 denotes inlet state and index 2 denotes end state after isentropic compression (Figure 2). 


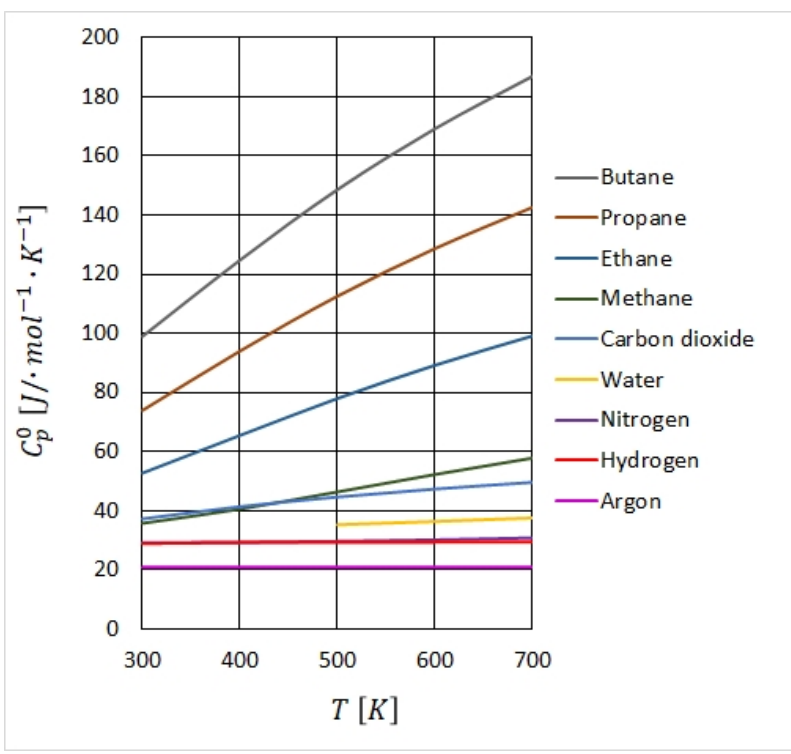

FiguRE 3. Dependence of $C_{p}^{0}$ on temperature for several common used gases 9 .

\section{Methods of CAlCulation OF ISENTROPIC PROCESS AND ISENTROPIC EFFICIENCY}

Before we come to the description of the solution of each type of calculation, it will be useful to mention several methods of solving the isentropic process.

- In the simplest case, we can assume that we are compressing the ideal gas. The calculation will be very easy and very inaccurate.

- Entropy was often read from diagrams or tables in the past. The accuracy was limited by the size of the diagram. However, this method is completely inappropriate for calculating gaseous mixtures and cannot be used on PC.

- Another of the approximate methods of calculating the isentropic process is the method based on the relations valid for the ideal gas, i.e. on the Poisson equations (10), (11) and (12). Isentropic exponent is calculated using of real gas EOS and general relationships.

$$
\begin{gathered}
k_{p, V}=-\frac{V}{p} \frac{C_{p}}{C_{V}}\left(\frac{\partial p}{\partial V}\right)_{T}, \\
k_{p, T}=\frac{1}{1-\frac{p}{C_{p}}\left(\frac{\partial V}{\partial T}\right)_{p}}, \\
k_{T, V}=1+\frac{V}{C_{V}}\left(\frac{\partial p}{\partial T}\right)_{V} .
\end{gathered}
$$

Usually we do not know all parameters of end point of isentropic change (Figure 2p. Thus we have to use value of isentropic exponent calculated for mean pressure and temperature. We estimate parameters in the point of interest from the behavior of the ideal gas. This method is suitable for PC and is appropriate for calculation of gas mixtures. Even in this case, we do not have enough accurate results.

As an illustration of the inappropriate use of the relationships derived for the ideal gas in combination with the isentropic exponent calculated by means of the real gas equation of state from the relationship (15), the calculation of the isentropic change from the relation can serve calculation of isentropic enthalpy from equation

$$
\Delta H_{s}=p_{1} V_{1} \frac{k_{p, V}}{k_{p, V}-1}\left[\left(\frac{p_{2}}{p_{1}}\right)^{\frac{k_{p, V}-1}{k_{p, V}}}-1\right]
$$

There are a number of other methods described in literature but further we focus only on methods which use the solution of a system of nonlinear equations, one of which is always a chosen real gas equation of state and one the requirement for zero change of entropy in transition from state 1 to 2 (Figure 2).

The following paragraphs provide algorithms of solution for individual types of calculations. In their design, particular emphasis was placed on working reliably and quickly. For every combination of input parameters the individual algorithm for solution of system of nonlinear equations was designed. Important parts of every algorithm are relations for estimation of the initial approximations. In all cases, the calculation can be divided into a part independent of the real gas EOS and the equation for the $C_{p}^{0}=C_{p}^{0}(T)$ of the ideal gas and a part dependent on these equations. The suitable method for solution of this system is Newton's method.

Functions $F_{i}$ that are used in the next detailed description of algorithms are shown in Table 3. To construct the Jacobi matrix of functions $F_{i}$ that we need for solution by the Newton method, we need to know the partial derivatives of $F_{i}$. These derivatives are shown in Table 4 . The abovementioned derivatives can also be obtained by numerical derivation of functions $F_{i}$. In Table 5 there are relations for calculation of entropy and enthalpy of real gas and relations for calculation of dimensionless quantities $Q$ [10].

Any real gas EOS can be chosen for the following calculations. Dimensionless quantities Q for chosen EOS can be found in literature or can be derived from relations described in Table 5 .

\section{Calculations Based on the ReAl GAS EOS}

In practical calculations, we need to find unknown parameters when we know the initial state and two parameters of discharge state. The combination of input and output parameters is shown in Table 1.

In case that isentropic efficiency has value one i.e. $\eta_{s}=1$ it is true $p_{2 s}=p_{2 a}, T_{2 s}=T_{2 a}$ and $\Delta H_{s}=\Delta H_{a}$, then items 1,2 and 3 from Table 1 show combination of input and output parameters of isentropic change of thermodynamic state see Table 2 . 


\begin{tabular}{lll}
\hline & Input & Output \\
\hline 1 & $p_{1}, T_{1}, p_{2 s}$ & $T_{2 s}, \Delta H_{s}$ \\
2 & $p_{1}, T_{1}, T_{2 s}$ & $p_{2 s}, \Delta H_{s}$ \\
3 & $p_{1}, T_{1}, \Delta H_{s}$ & $p_{2 s}, T_{2 s}$ \\
\hline
\end{tabular}

TABLE 1. Combination of output and input parameters for isentropic process.

\begin{tabular}{lll}
\hline & Input & Output \\
\hline 1 & $p_{1}, T_{1}, p_{2 a}, \eta_{s}$ & $T_{2 a}, \Delta H_{a}$ \\
2 & $p_{1}, T_{1}, p_{2 a}, \Delta H_{a}$ & $T_{2 a}, \eta_{s}$ \\
3 & $p_{1}, T_{1}, T_{2 a}, \eta_{s}$ & $p_{2 a}, \Delta H_{a}$ \\
4 & $p_{1}, T_{1}, T_{2 a}, \Delta H_{a}$ & $p_{2 a}, \eta_{s}$ \\
5 & $p_{1}, T_{1}, p_{2 a}, T_{2 a}$ & $\Delta H_{a}, \eta_{s}$ \\
\hline
\end{tabular}

TABLE 2. Combination of output and input parameters.

\subsection{Example of The SOlution BY NEWTON'S METHOD}

All combinations of parameters are mentioned in [4]. For one of them we will show the solution. We will compute isentropic temperature $T_{2}=T_{2 s}$ and isentropic change in enthalpy $\Delta H=\Delta H_{s}$ from known parameters $p_{1}, T_{1}$ and $p_{2}$ (Figure 2). It is variant 1 from Table1 In this case we assume that the isentropic efficiency is equal to one $\eta_{s}=1$. Since this is the isentropic process, the original system of four equations is now simplified to only two equations $F_{1}=0$, $F_{2}=0$.

When we use general equation of isentropic process $S_{1}-S_{2}=0$ (Figure 2) and general expression of real gas EOS we obtain the system of two nonlinear equations for two variables $T_{2}$ and $\rho_{2}$

$$
\begin{gathered}
F_{1}=S_{1}\left(T_{1}, \rho_{1}\right)-S_{2 s}\left(T_{2 s}, \rho_{2 s}\right)=0, \\
F_{2}=\frac{p_{2 s}}{\rho_{2 s} R T_{2 s}}-z_{2 s}\left(T_{2 s}, \rho_{2 s}\right)=0 .
\end{gathered}
$$

This system of equations will be solved by Newton's method. Because it is known and very often used method, we will show only an matrix notation of linear equations for increments in $\Delta T$ and $\Delta \rho$

$$
\left[\begin{array}{ll}
\frac{\partial F_{1}}{\partial T_{2 s}} & \frac{\partial F_{1}}{\partial \rho_{2 s}} \\
\frac{\partial F_{2}}{\partial T_{2 s}} & \frac{\partial F_{2}}{\partial \rho_{2 s}}
\end{array}\right] \cdot\left[\begin{array}{c}
\Delta T \\
\Delta \rho
\end{array}\right]=\left[\begin{array}{l}
-F_{1} \\
-F_{2}
\end{array}\right]
$$

Desired parameters $T_{2}$ and $\rho_{2}$ can be obtained from relationships

$$
\begin{gathered}
T_{2 s}^{(k+1)}=T_{2 s}^{(k)}+\Delta T, \\
\rho_{2 s}^{(k+1)}=\rho_{2 s}^{(k)}+\Delta \rho .
\end{gathered}
$$

The calculation is completed by fulfilling of the requiremen $\max (|\delta T| ;|\delta \rho|) \leq \epsilon$ where $\delta T$ and $\delta \rho$ are relative deviations and $\epsilon$ is chosen value.

Detail description of solution for this example: For inlet condition $p_{1}, T_{1}$ we determine values $S_{1}$ and $H_{1}$ from real gas EOS next we calculate $C_{V}^{0}$

$$
C_{p}^{0}=C_{p}^{0}\left(T_{1}, \vec{x}\right), \quad C_{V}^{0}=C_{p}^{0}-R
$$

Next step is solution of system of equations $F_{1}=0$, $F_{2}=0$ by using of Newton's method. Initial approximations are relations valid for ideal gas

$$
\begin{gathered}
T_{2 s}^{(0)}=T_{1}\left(\frac{p_{2 s}}{p_{1}}\right)^{\frac{C_{p}^{0}-C_{V}^{0}}{C_{P}^{0}}} \\
\rho_{2 s}^{(0)}=\frac{p_{2 s}}{R T_{2 s}^{(0)}}
\end{gathered}
$$

As the results we obtain $T_{2 s}$ and $\rho_{2 s}$. From general form of EOS we obtain compressibility factor in point $2_{s}$ (Figure 2 )

$$
z_{2 s}=\frac{p_{2 s}}{R T_{2 s} \rho_{2 s}}
$$

and enthalpy

$$
H_{2 s}=H_{2 s}\left(T_{2 s}, \rho_{2 s}, \vec{x}\right) .
$$

Finally we calculate change in enthalpy

$$
\Delta H=H_{2}-H_{1}
$$

where $H_{2}=H_{2 s}$.

\subsection{CAlculation of $T_{2 s}, \Delta H_{a}$ FROM} KNOWN PARAMETERS $p_{1}, T_{1}, p_{2 a}$ AND $\eta_{s}$ (FIGURE 2, TABLE 2 - ITEM 1)

We solve the system of equations $F_{1}=0, F_{2}=0$, $F_{4}=0$ and $F_{5}=0$ for unknown parameters $T_{2 s}, \rho_{2 s}$, $T_{2 a}, \rho_{2 a}$.

Initial approximations are

$$
\begin{gathered}
T_{2 s}^{(0)}=T_{1}\left(\frac{p_{2 a}}{p_{1}}\right)^{\frac{C_{p}^{0}-C_{V}^{0}}{C_{P}^{0}}} \\
\rho_{2 s}^{(0)}=\frac{p_{2 a}}{R T_{2 s}^{(0)}} \\
T_{2 a}^{(0)}=\frac{T_{2 s}^{(0)}-T_{1}}{\eta_{s}}+T_{1}, \\
\rho_{2 a}^{(0)}=\frac{p_{2 a}}{R T_{2 a}^{(0)}}
\end{gathered}
$$

where $C_{p}^{0}$ is

$$
C_{p}^{0}=C_{p}^{0}\left(T_{1}, \vec{x}\right), \quad C_{V}^{0}=C_{p}^{0}-R
$$

Change in enthalpy $\Delta H_{a}$ we obtain from equation

$$
\Delta H_{a}=H_{2 a}-H_{1}
$$

where

$$
H_{2 a}=f\left(T_{2 a}, \rho_{2 a}, \vec{x}\right) .
$$


7.3. Calculation of $T_{2 a}, \eta_{s}$ FROM KNOWN PARAMETERS $p_{1}, T_{1}, p_{2 a}$ AND $\Delta H_{a}$ (FIGURE 2, TABLE 2 - ITEM 2)

We solve the system of four equations $F_{1}=0, F_{2}=0$, $F_{5}=0, F_{6}=0$ for unknown parameters $T_{2 s}, \rho_{2 s}, T_{2 a}$, $\rho_{2 a}$.

Initial approximation $T_{2 s}^{(0)}$ we obtain from Eq. (30), $\rho_{2 s}^{(0)}$ from Eq. 31

$$
T_{2 s}^{(0)}=T_{1}+\frac{\Delta H_{a}}{C_{p}^{0}}
$$

and $\rho_{2 a}^{(0)}$ from Eq. $(33)$.

Isentropic efficiency $\eta_{s}$ we calculate from Eq. (2), where enthalpy $H_{1}$ we know from calculation in initial point 1 and $H_{2 a}=f\left(T_{2 a}, \rho_{2 a}, \vec{x}\right)$.

\subsection{Calculation of $p_{2 a}, \Delta H_{a}$ From KnOWN} PARAMETERS $p_{1}, T_{1}, T_{2 a}$ AND $\eta_{s}$ (Figure 2, table 2 - ITEM 3)

Calculation is based on solving of three equations $F_{1}=0, F_{4}=0$ and $F_{8}=0$ for unknown parameters $T_{2 s}, \rho_{2 s}$ and $\rho_{2 a}$.

The first approximations are

$$
\begin{gathered}
T_{2 s}^{(0)}=\eta_{s}\left(T_{2 a}-T_{1}\right)+T_{1}, \\
\rho_{2 s}^{(0)}=\rho_{1}\left(\frac{T_{2 s}}{T_{1}}\right)^{\frac{C_{V}^{0}}{C_{p}^{0}-C_{V}^{0}}}, \\
\rho_{2 a}^{(0)}=\frac{T_{2 s}^{(0)} \rho_{2 s}^{(0)}}{T_{2 a}},
\end{gathered}
$$

where $C_{p}^{0}$ see Eq. 34 .

After every step of the Newton's method the k-th approximation of the compressibility factor $z_{2 a}^{(k)}$ is found from real gas equation of state and then the $\mathrm{k}$-th approximation of the pressure $p_{2 a}^{(k)}$ is calculated from equation

$$
p_{2 a}^{(k)}=z_{2 a}^{(k)} R T_{2 a} \rho_{2 a}^{(k)} .
$$

From equality $p_{2 s}=p_{2 a}$ (see figure 2), we can calculate

$$
z_{2 s}^{(k)}=\frac{z_{2 a}^{(k)} T_{2 a} \rho_{2 a}^{(k)}}{T_{2 s}^{(k)} \rho_{2 s}^{(k)}}
$$

Change in enthalpy $\Delta H_{a}$ is determined from Eq. (1), where $H_{2 a}=f\left(T_{2 a}, \rho_{2 a}, \vec{x}\right)$.

\subsection{Calculation of $p_{2 a}, \eta_{s}$ From KnOWN PARAMETERS $p_{1}, T_{1}, T_{2 a}$ AND $\Delta H_{a}$ (FIGURE 2, TABLE 2 - ITEM 4)}

To calculate unknown parameters $T_{2 s}, \rho_{2 s}$ and $\rho_{2 a}$ system of three equations $F_{1}=0, F_{6}=0$ and $F_{8}=0$ is solved.

For the first approximation of temperature we choose $T_{2 s}^{(0)}=T_{2 a}$. Density $\rho_{2 s}^{(0)}$ we obtain from equation $40, \rho_{2 a}^{(0)}=\rho_{2 s}^{(0)}$ and $z_{2 a}^{(0)}=z_{2 s}^{(0)}=1$. After every

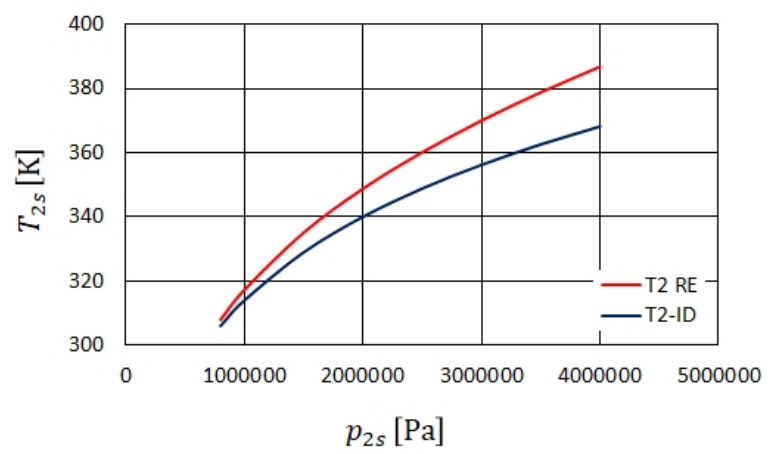

FiguRE 4. Ideal gas temperature $T_{2 s}$ calculated from Eq. (30) - blue curve and real gas temperature $T_{2 s}-$ red curve. Inlet conditions propane $p_{1}=550000 \mathrm{~Pa}$, $T_{1}=293.15 \mathrm{~K}$.

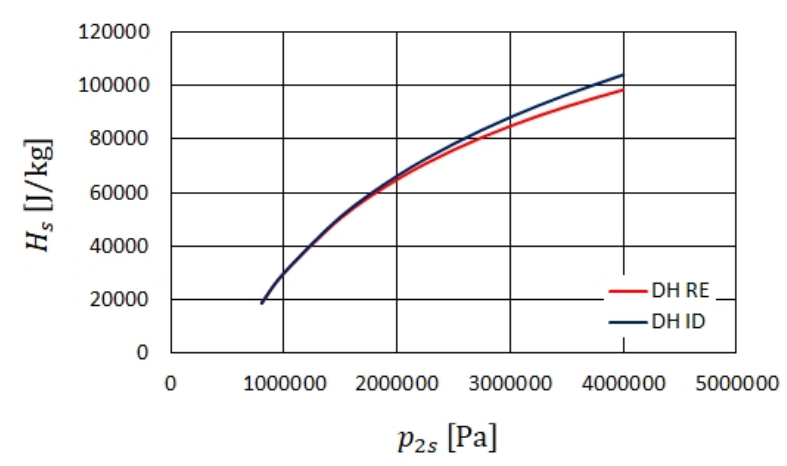

FiguRE 5. Ideal gas isentropic work $\Delta H_{s}$ calculated from Eq. 43 - blue curve and real gas isentropic work $\Delta H_{s}-$ red curve. Inlet conditions propane $p_{1}=$ $550000 \mathrm{~Pa}, T_{1}=293.15 \mathrm{~K}$.

step of Newton's method compressibility factors $z_{2 a}^{(k)}$ and $z_{2 s}^{(k)}$ are calculated similar way like in previous case i.e. by solution of real gas EOS and by using of Equations (41) a 421.

Finally isentropic efficiency $\eta_{s}$ we obtain from Eq. (2), where $H_{1}$ is known from calculation of thermodynamic properties in point 1 (figure 2) and $H_{2 s}=f\left(T_{2 s}, \rho_{2 s}, \vec{x}\right)$.

\subsection{Calculation of $\Delta H_{a}, \eta_{s}$ From KNOWN PARAMETERS $p_{1}, T_{1}, p_{2 a}$ AND $T_{2 a}$ (Figure 2, table 2 - ITEM 5)}

To calculate unknown parameters $T_{2 s}, \rho_{2 s}$ and $\rho_{2 a}$ system of three equations $F_{1}=0, F_{2}=0$ and $F_{5}=0$ is solved.

First approximation of temperature $T_{2 s}^{(0)}$ we obtain from Eq. 30$), \rho_{2 s}^{(0)}$ from Eq 31 and $\rho_{2 a}^{(0)}$ from Eq. 33.

Change in enthalpy $\Delta H_{a}$ we calculate by using of Eq. (1), where $H_{2 a}=f\left(T_{2 a}, \rho_{2 a}, \vec{x}\right)$. Isentropic efficiency $\eta_{s}$ we finally obtain from Eq. (2), where $H_{2 s}=f\left(T_{2 s}, \rho_{2 s}, \vec{x}\right)$. 


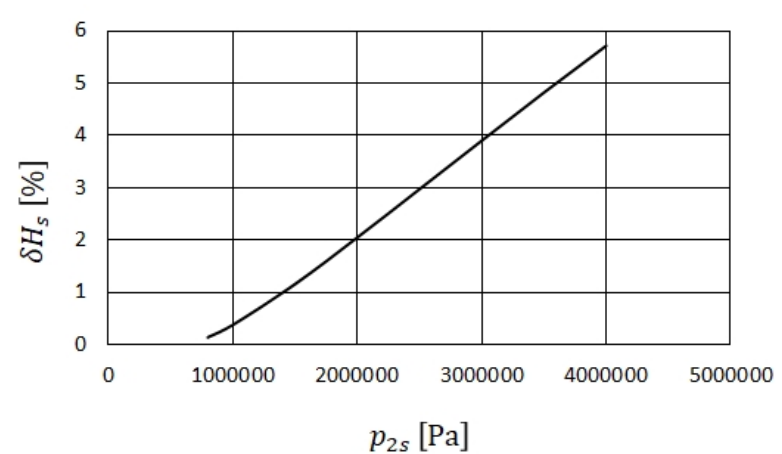

FiguRE 6. Relative deviation of isentropic work calculated for real and ideal gas.

\section{EXAMPLE}

As an example the calculation of temperature and isentropic work after isentropic change was chosen. In this example compression of propane from state 1 to state $2_{s}$ (see Figure 2) is calculate by two methods. In the first case above described method based on the real gas is used and in the second case method based on an ideal gas. Parameters in point 1 are pressure $p_{1}=550000 \mathrm{~Pa}$, temperature $T_{1}=293.15 \mathrm{~K}$. The third parameter is gas pressure $p_{2 s}$ which varies in the interval 800000 to $2500000 \mathrm{~Pa}$. For calculation of real gas isentropic change the method described in paragraph 7.1 was used. This method is equivalent to method of paragraph 7.2 when $\eta_{s}=1$.

For calculation of temperature $T_{2 s}$ of ideal gas the Equation (30) was for $p_{2 a}=p_{2 s}=p_{2}$. Isentropic change of enthalpy was calculated from Eq. 18 in form

$$
\Delta H_{s}=z_{1} R T_{1} \frac{k}{k-1}\left[\left(\frac{p_{2}}{p_{1}}\right)^{\frac{k-1}{k}}-1\right],
$$

where $z_{1}$ and $k$ were calculated from BWR EOS. Results of all calculations are on Figures 4, 5 and 6 .

\section{Conclusion}

The methods described in this contribution exactly solved problem of calculation of the isentropic efficiency for various input parameters. In case that efficiency is equal zero above described procedures are suitable for calculation of isentropic process. The above described methods advantageously use the dimensionless variables defined in Table 5 and real gas EOS.

It is evident from the work that some approximate procedures can lead to inaccurate results.

Methods described here together with method described in [6] and in [7] and suitable real gas EOS form the basic tools for thermodynamic calculations of compressors or others flow machines.

\section{LIST OF SYMBOLS \\ $T$ thermodynamic temperature $[\mathrm{K}]$}

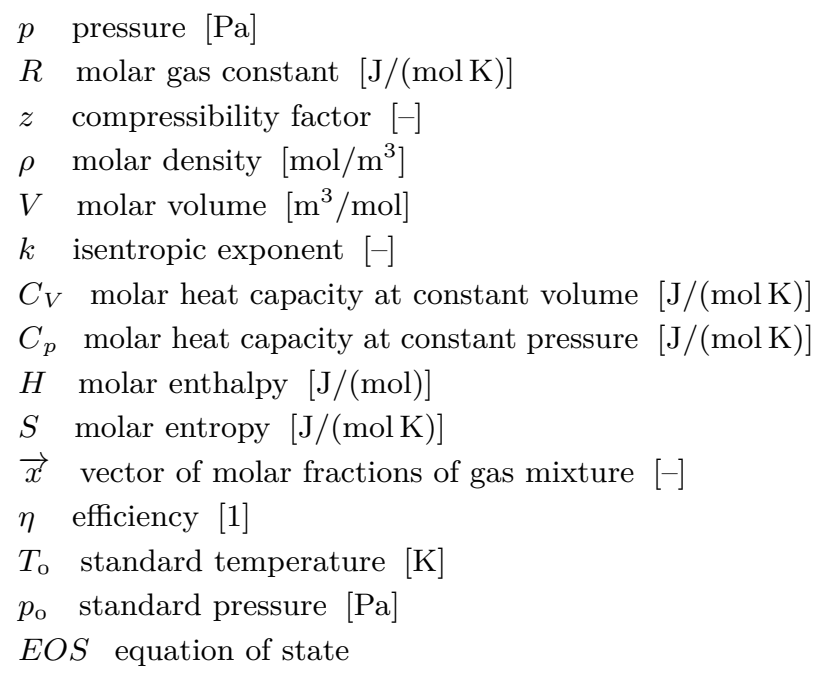

\section{INDEXES:}

$s$ isentropic

a actual

1 inlet, input

2 discharge, output

id ideal gas

0 thermodynamic quantities of ideal gas (upper index)

\section{REFERENCES}

[1] D. A. Kouremenos, X. K. Kakatsios. The three isentropic exponents of dry steam. Forschung auf dem Gebiete des Ingenieurwesens 51:117-122, 1985. DOI:10.1007/BF02558416

[2] D. Misárek. Turbokompresory (Turbocompressors). SNTL, 1963. In Czech.

[3] K. H. Lüdtke. Process Centrifugal Compressors. Springer, Berlin, Heidelberg, 2004. DOI:10.1007/978-3-662-09449-5_9.

[4] J. Oldřich, J. Novák, A. Malijevský. Adiabatický děj v technické praxi, (Adiabatic pro-cess in technical practice). Strojírenství 40(2):69-76, 1990. In Czech.

[5] J. M. Schultz. The polytropic analysis of centrifugal compressors. Journal of Engineering for Power 84:69, 1962. DOI:10.1115/1.3673381.

[6] J. Oldřich. Advanced polytropic calculation method of centrifugal compressor. 2010. DOI:10.1115/IMECE2010-40931

[7] J. Oldřich. Calculation of the isothermal efficiency of a cen-trifugal compressor compressing the real gas. In Proceedings of conference "Turbostroje 2016". Plzeň, Czech Republic, 2016.

[8] B. Eckert. Axialkompressoren und Radialkompressoren. Springer-Verlag, Berlin, 1953. DOI:10.1007/978-3-642-52720-3

[9] P. J. Linstrom, W. G. Mallard. NIST Chemistry WebBook, NIST Standard Refer-ence Database Number 69. DOI:10.18434/T4D303.

[10] J. P. Novák, A. Malijevský, J. Pick. Použití bezrozměrných veličin při výpočtu termo-dynamických funkcí, (Use of dimensionless quan-tities for calculating of thermodynamic functions from equations of state). Chemické listy 73:1178-82, 1979. In Czech. 


$$
\begin{aligned}
& F_{1}=S_{1}\left(T_{1}, \rho_{1}\right)-S_{2}\left(T_{2 s}, \rho_{2 s}\right) \\
& F_{2}=\frac{p_{2 s}}{\rho_{2 s} R T_{2 s}}-z_{2}\left(T_{2 s}, \rho_{2 s}\right) \\
& F_{3}=\Delta H_{s}-\left[H_{2}\left(T_{2 s}, \rho_{2 s}\right)-H_{1}\left(T_{1}, \rho_{1}\right)\right] \\
& F_{4}=\eta_{s}\left[H_{2}\left(T_{2 a}, \rho_{2 a}\right)-H_{1}\left(T_{1}, \rho_{1}\right)\right]-\left[H_{2}\left(T_{2 s}, \rho_{2 s}\right)-\right. \\
& \left.F_{5}=\frac{p_{2 s}}{\rho_{2 a} R T_{2 a}}-z_{2}\left(T_{2 a}, \rho_{2 a}\right)\right] \\
& F_{6}=\left[H_{2}\left(T_{2 a}, \rho_{2 a}\right)-H_{1}\left(T_{1}, \rho_{1}\right)\right]-\Delta H_{a} \\
& F_{7}=\eta_{S} \Delta H_{a}-\left[H_{2}\left(T_{2 s}, \rho_{2 s}\right)-H_{1}\left(T_{1}, \rho_{1}\right)\right] \\
& F_{8}=z_{2}\left(T_{2 a}, \rho_{2 a}\right) T_{2 a} \rho_{2 a}-z_{2}\left(T_{2 s}, \rho_{2 s}\right) T_{2 s} \rho_{2 s}
\end{aligned}
$$

TABLE 3. Functions $F_{i}$.

$$
\begin{aligned}
\frac{\partial F_{1}}{\partial T_{2 s}} & =\frac{R}{T_{2 s}}\left(1+2 Q_{U 2 s}+Q_{C 2 s}\right)-\frac{\sum x_{i} C_{p i}^{0}\left(T_{2 s}\right)}{T_{2 s}} \\
\frac{\partial F_{1}}{\partial \rho_{2 s}} & =\frac{R Q_{T 2 s}}{\rho_{2 s}} \\
\frac{\partial F_{2}}{\partial T_{2 s}} & =-\frac{Q_{T 2 s}}{T_{2 s}} \\
\frac{\partial F_{2}}{\partial \rho_{2 s}} & =-\frac{Q_{\rho 2 s}}{\rho_{2 s}} \\
\frac{\partial F_{2}}{\partial T_{2 a}} & =\frac{\partial F_{2}}{\partial \rho_{2 a}}=\frac{\partial F_{6}}{\partial T_{2 s}}=\frac{\partial F_{6}}{\partial \rho_{2 s}}=\frac{\partial F_{7}}{\partial \rho_{2 a}}=0 \\
\frac{\partial F_{3}}{\partial T_{2 s}} & =\frac{\partial F_{4}}{\partial T_{2 s}}=\frac{\partial F_{7}}{\partial T_{2 s}}=-R\left(Q_{T 2 s}-2 Q_{U 2 s}-Q_{C 2 s}-\right. \\
\frac{\partial F_{3}}{\partial \rho_{2 s}}= & \frac{\partial F_{4}}{\partial \rho_{2 s}}=\frac{\partial F_{7}}{\partial \rho_{2 s}}=\frac{R T_{2 s}\left(Q_{T 2 s}-Q_{\rho 2 s}\right)}{\rho_{2 s}} \\
\frac{\partial F_{4}}{\partial T_{2 a}}=\eta_{s}\left[R\left(Q_{T 2 a}-2 Q_{U 2 a}-Q_{C 2 a}-1\right)+\right. & \left.+\sum x_{i} C_{p i}^{0}\left(T_{2 s}\right)\right]
\end{aligned}
$$$$
\frac{\partial F_{4}}{\partial \rho_{2 a}}=\eta_{s} \frac{R T_{2 a}\left(Q_{\rho 2 a}-Q_{T 2 a}\right)}{\rho_{2 a}}
$$$$
\frac{\partial F_{1}}{\partial T_{2 a}}=\frac{\partial F_{1}}{\partial \rho_{2 a}}=\frac{\partial F_{5}}{\partial T_{2 s}}=\frac{\partial F_{5}}{\partial \rho_{2 s}}=0
$$$$
\frac{\partial F_{5}}{\partial T_{2 a}}=-\frac{Q_{T 2 a}}{T_{2 a}}
$$$$
\frac{\partial F_{5}}{\partial \rho_{2 a}}=-\frac{Q_{\rho 2 a}}{\rho_{2 a}}
$$$$
\frac{\partial F_{6}}{\partial T_{2 a}}=R\left(Q_{T 2 a}-2 Q_{U 2 a}-Q_{C 2 a}-1\right)+
$$$$
+\sum x_{i} C_{p i}^{0}\left(T_{2 s}\right)
$$$$
\frac{\partial F_{6}}{\partial \rho_{2 a}}=\frac{R T_{2 a}\left(Q_{\rho 2 a}-Q_{T 2 a}\right)}{\rho_{2 a}}
$$$$
\frac{\partial F_{8}}{\partial T_{2 s}}=-\rho_{2 s} Q_{T 2 s}
$$$$
\frac{\partial F_{8}}{\partial \rho_{2 s}}=-T_{2 s} Q_{\rho 2 s}
$$$$
\frac{\partial F_{8}}{\partial T_{2 a}}=\rho_{2 a} Q_{T 2 a}
$$$$
\frac{\partial F_{8}}{\partial \rho_{2 a}}=T_{2 a} Q_{\rho 2 a}
$$

TABLE 4. Derivative of functions $F_{i}$. 


$$
\begin{aligned}
& z=z(T, \rho)=\frac{p}{R T \rho} \\
& S(T, \rho, \vec{x})=\sum x_{1}\left[S_{i}^{0}\left(T_{0}\right)+\int_{T_{0}}^{T} \frac{C_{p i}^{0}(T)}{T} d T\right]- \\
& -R \ln \frac{z R T \rho}{p_{0}}-R \sum x_{i} \ln x_{i}+R\left(\ln z-Q_{F}-Q_{U}\right) \\
& H(T, \rho, \vec{x})=\sum x_{1}\left[H_{i}^{0}\left(T_{0}\right)+\int_{T_{0}}^{T} C_{p i}^{0}(T) d T\right]+ \\
& +R T\left(z-1-Q_{U}\right) \\
& Q_{\rho}=z+\rho\left(\frac{\partial z}{\partial \rho}\right)_{T} \\
& Q_{F}=\int_{0}^{\rho}(z-1) d(\ln \rho) \\
& Q_{T}=z+T\left(\frac{\partial z}{\partial T}\right)_{\rho} \\
& Q_{U}=T\left(\frac{\partial Q_{F}}{\partial T}\right)_{\rho} \\
& Q_{C}=T^{2}\left(\frac{\partial^{2} Q_{F}}{\partial T^{2}}\right)_{\rho}
\end{aligned}
$$

TABLE 5. Relations for calculation of entropy and enthalpy of real gas and relations for calculation of dimensionless quantities $Q$ [10]. 